\title{
The domination number of plane triangulations
}

\author{
Simon Špacapan*
}

October 5, 2018

\begin{abstract}
We introduce a class of plane graphs called weak near-triangulations, and prove that this class is closed under certain graph operations. Then we use the properties of weak near-triangulations to prove that every plane triangulation on $n>6$ vertices has a dominating set of size at most $17 n / 53$. This improves the bound $n / 3$ obtained by Matheson and Tarjan.
\end{abstract}

Key words: triangulation, dominating set

AMS subject classification (2010): 05C10, 05C69

\section{Introduction}

A dominating set in a graph $G=(V, E)$ is a set $D \subseteq V$, such that every vertex in $V \backslash D$ is adjacent to a vertex in $D$. The domination number of $G$, denoted as $\gamma(G)$, is the minimum size of a dominating set in $G$. A plane triangulation is a connected simple plane graph $G$, such that every face of $G$ is triangular. A plane graph $G$ is a near-triangulation, if $G$ is 2-connected and every face of $G$ is triangular, except possibly the unbounded face.

Matheson and Tarjan conjectured in [11 that every sufficiently large plane triangulation has a dominating set of cardinality at most $n / 4$, where $n$ is the number of vertices of the triangulation. They proved that the domination number of every neartriangulation, and therefore also every triangulation, is at most $n / 3$.

Theorem 1.1 [11] Every near-triangulation has a 3-coloring such that each color class is a dominating set.

We mention that Theorem 1.1 provides the only known upper bound for $\gamma(G) / n$ in the class of plane triangulations. Several results for sublasses of plane triangulations are known. For example, the conjecture of Matheson and Tarjan was confirmed in [7] for plane triangulations with maximum degree 6. In [9] the authors improve the bound

\footnotetext{
${ }^{*}$ University of Maribor, FME, Smetanova 17, 2000 Maribor, Slovenia. e-mail: simon.spacapan @um.si.
} 
$n / 4$ obtained in [7], by proving that there exists a constant $c$ such that $\gamma(G) \leq n / 6+c$ for every plane triangulation $G$ with maximum degree 6 . Another result is given in [14] where the authors prove that $\gamma(G) \leq \max \left\{\left\lceil\frac{2 n}{7}\right\rceil,\left\lfloor\frac{5 n}{16}\right\rfloor\right\}$ for every 4-connected plane triangulation $G$. The domination number of outerplanar triangulations is considered in [1] and [15], where the authors independently prove that an outerplaner triangulation with $n$ vertices and $t$ vertices of degree 2 has a dominating set of cardinality at most $(n+t) / 4$. This result is further improved in [8].

Theorem 1.1 was extended to tringulations on the projective plane, the torus and the Klein bottle in [13] and in [6]. It is proved that every triangulation on any of these surfaces has a dominating set of cardinality at most $n / 3$. These results are further generalized in [4], where it is proved that every triangulation on a closed surface has a dominating set of cardinality at most $n / 3$.

We also mention that the domination number of planar graphs with small diameter is studied in [10], 5] and [3]. It is proved that every sufficiently large planar graph with diameter 3 has domination number at most 6 .

We approach the problem of finding the smallest constant $c$, such that $\gamma(G) \leq c n$, for every sufficiently large plane triangulation. In the following section we introduce the class of weak near-triangulations. We define reducibility of a weak near-triangulation, and prove that every weak near-triangulation $G$ is reducible, except if all blocks of $G$ are outerplaner or have exactly 6 vertices. This result is then used to prove that every plane triangulation on $n>6$ vertices has a dominating set of cardinality at most $17 \mathrm{n} / 53$.

\section{The domination number of triangulations}

We refer the reader to [2] and [12] for a complete overwiev of definitions and terminology that we use. In this article we consider only simple graphs with no multiple edges or loops. Let $G$ be a plane graph. An edge $e$ of $G$ is incident to a face $F$ of $G$ if $e$ is contained in the boundary of $F$. Similarly we define incidence of a verex $x$ and a face $F$. Vertices and edges incident to the unbounded face of $G$ are called external vertices and external edges, respectively. If a vertex (an edge) is not an external vertex (edge), then it is called an internal vertex (internal edge). A triangle is a cycle on three vertices. If $a, b$ and $c$ are vertices of a triangle, we denote this triangle by $a b c$, and we say that $a, b$ and $c$ are contained in the triangle $a b c$. A facial triangle of a plane graph $G$ is a triangle whose interior is a face of $G$. A face bounded by a triangle is called a triangular face. A connected plane graph $G$ is a triangulation if all faces of $G$ are triangular, and $G$ is a near-triangulation if it is 2-connected and every bounded face of $G$ is triangular. A block of a graph $G$ is a maximal connected subgraph of $G$ without a cutvertex.

Definition 2.1 A plane graph $G$ is a weak near-triangulation (WNT) if every bounded face of $G$ is triangular and every vertex of $G$ is contained in a triangle.

We note that an empty graph is a weak near-triangulation, and that in the above definition there are no assumptions about the connectivity of $G$, hence $G$ may be 


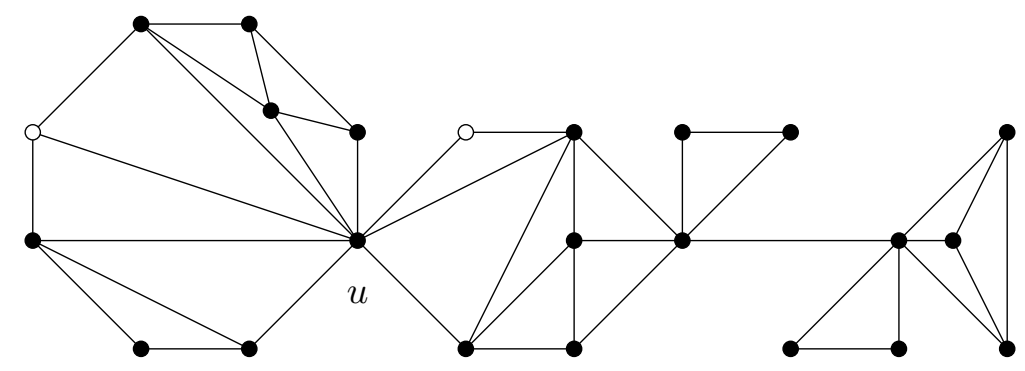

Figure 1: A weak near-triangulation $G$.

disconnected. Note also that the definition is equivalent to the following: $G$ is a weak near-triangulation if every bounded face of $G$ is triangular and every vertex of $G$ is contained in a facial triangle.

In 2] (see Lemma 4.2.2., p. 91, and Lemma 4.2.6, p. 93) the following two results are given.

Lemma 2.2 Let $G$ be a plane graph and e an edge of $G$. If e lies on a cycle $C \subseteq G$, then $e$ is incident to exactly one face $F$ of $G$, such that $F$ is contained in the interior of $C$.

Lemma 2.3 In a 2-connected plane graph, every face is bounded by a cycle.

We use Lemma 2.2 and 2.3 to prove the following.

Lemma 2.4 Every block of a weak near-triangulation is either a near-triangulation or a $K_{2}$.

Proof. Let $B$ be a block of a weak near-triangulation $G$. Since every vertex of $G$ is contained in a triangle of $G$, we find that $B$ has more than one vertex. Suppose that $B$ is a block on more than two vertices. Then $B$ is 2 -connected, and so by Lemma 2.3 every face of $B$ is bounded by a cycle. Let $F$ be a bounded face of $B$ and $C$ the cycle bounding $F$ ( $F$ lies in the interior of $C$ ). To prove that $B$ is a near-triangulation, we have to prove that $F$ is triangular.

Let $e=u v$ be any edge of $C$. Since $C$ is a cycle of $G$ we find, by Lemma 2.2, that there is a face $F^{\prime}$ of $G$ contained in the interior of $C$ incident to $e$. Since $F^{\prime}$ is a bounded face of $G$, it has to be triangular (by the definition of a weak near-triangulation). If $F^{\prime}=F$ we are done. Suppose that $F^{\prime} \neq F$, and suppose that $F^{\prime}$ is bounded by the triangle $u v x$. Then $x$ is not in $B$, for otherwise $F$ and $F^{\prime}$ are faces of $B$ contained in the interior of $C$, both incident to $e$ (contradictory to Lemma 2.2). So $x \notin B$ and therefore $B$ is not a maximal connected subgraph of $G$ without a cutvertex (we may add $x$ to $B$ ), a contradiction.

Observe also that every endblock (leaf block) of a weak near-triangulation is a near-triangulation. 
Lemma 2.5 Every weak near-triangulation has a 3-coloring such that each color class is a dominating set.

Proof. Let $G$ be a weak near-triangulation. Delete all bridges of $G$ and call the obtained graph $G^{\prime}$. Every block of $G^{\prime}$ is a near-triangulation, and two distinct blocks have at most one common vertex. To obtain the desired 3-coloring of a connected component of $G^{\prime}$ we use induction on number of blocks. In induction step we delete an endblock (except the cutvertex) and color the obtained graph with 3 colors according to the induction hypothesis. Then use Theorem 1.1 to obtain a coloring of the deleted endblock, if needed permute the color classes in the endblock (so that the cutvertex gets the color that it already has).

A straightforward corollary is the following.

Corollary 2.6 Every weak near-triangulation on $n$ vertices has domination number at most $n / 3$.

For a plane graph $G$ and $X \subseteq V(G)$ we denote by $G-X$ the graph obtained from $G$ by deleting the vertices in $X$ and all edges incident to a vertex in $X$. If $X=\{u\}$ is a singleton, we write $G-u$ instead of $G-\{u\}$. If $H$ is a subgraph of a plane graph $G$, then we assume that the embedding of $H$ in the plane is that given by $G$. This in particular applies for $H=G-X$. Let $N[x]$ denote the set of vertices that are either adjacent or equal to $x, N[x]$ is called the closed neighborhood of $x$.

Definition 2.7 Let $G$ be a weak near-triangulation. We say that $G$ is reducible if there exists a set $D \subseteq V(G)$ and a vertex $x \in D$ with the following properties:

(i) $D \subseteq N[x]$

(ii) $|D| \geq 4$

(iii) $G-D$ is a weak near-triangulation.

The main result of this paper is the following theorem, the proof is postponed to the last section.

Theorem 2.8 Let $G$ be a weak near-triangulation. If $G$ has a block $B$, such that $B$ is not outerplanar and the order of $B$ is different from 6 , then $G$ is reducible.

Note that it is not possible to extend the above theorem to weak near-triangulations that have only outerplaner blocks and blocks on 6 vertices. We can see this by observing that an octahedron embedds in the plane as a triangulation, and it is not dominated by one vertex, and therefore also not reducible. Another example is given in Fig. 2 where the graph in case $(a)$ is not reducible. Observe also that every near-triangulation on $n$ vertices with domaination number $n / 3$ is not reducible, examples of such outerplaner near-triangulations are exhibited in [11. In fact, Theorem 2.8 and Corollary 2.6 imply the following. 
Corollary 2.9 Every near-triangulation that attains the bound $n / 3$ from Theorem 1.1 is outerplanar or has exactly 6 vertices.

Proof. By Theorem 2.8, every near-triangulation $G$ which is not outerplaner and has $n \neq 6$ vertices is reducible. So there exists a set $D$, such that $G-D$ is a weak neartriangulation, and $D$ is dominated by one vertex. The reduced graph $G-D$ has, according to Corollary 2.6, a dominating set containing at most $(n-|D|) / 3$ vertices. The result follows from $|D| \geq 4$.

It is easy to prove that every near-triangulation $G$ on 6 vertices has a vertex $x$, such that the closed neighborhood of $x$ contains all internal vertices of $G$. The reader may prove this by a case analysis: the outer cylce of $G$ has 5,4 or 3 vertices.

Corollary 2.10 Every plane triangulation on $n>6$ vertices has a dominating set of size at most $17 n / 53$.

Proof. Let $G$ be a plane triangulation on $n$ vertices. We apply Theorem 2.8 until the obtained graph $G^{\prime}$ is irreducible. More precisely, $G^{\prime}=G-\left(D_{1} \cup \ldots \cup D_{k}\right)$, where $D_{i}$ is a set of vertices in $G-\left(D_{1} \cup \ldots \cup D_{i-1}\right)$, such that there exists a vertex $x_{i} \in D_{i}$ with the property $D_{i} \subseteq N\left[x_{i}\right]$. Moreover, for all $i \leq k,\left|D_{i}\right| \geq 4$. By Theorem 2.8 , $G^{\prime}$ is a weak near-triangulation, and every block of $G^{\prime}$ is outerplanar or has exactly 6 vertices. If $G^{\prime}$ contains all three external vertices of $G$, then $G^{\prime}$ is a triangulation, and thus has exactly one block. If $G^{\prime}$ is a block of order 6 , then at least one reduction was done to get from $G$ to $G^{\prime}$. The vertices of $V(G) \backslash V\left(G^{\prime}\right)$ are dominated by a dominating set of size at most $\frac{1}{4}\left|V(G) \backslash V\left(G^{\prime}\right)\right|$. The block $G^{\prime}$ of order 6 is dominated by 2 vertices. It follows that $G$ has a dominating set of size at most $3 n / 10$. If $G^{\prime}$ is outerplaner, then it is a triangle. In this case $G$ has a dominating set of size at most $2 n / 7$.

Assume therefore that at least one external vertex of $G$ is not in $G^{\prime}$. We claim that every external vertex of $G^{\prime}$ is adjacent to a vertex in $V(G) \backslash V\left(G^{\prime}\right)$. Let $x$ be an external vertex of $G^{\prime}$. If $x$ is also an external vertex of $G$, the claim follows from the assumption. If $x$ is an internal vertex of $G$, then let $x y$ be an external edge of $G^{\prime}$ incident to $x$. Since $x$ is an internal vertex of $G$, the edge $x y$ is an internal edge of $G$, and so there are two facial triangles in $G$ incident to $x y$, and since $x y$ is an external edge of $G^{\prime}$, one of them is not a triangle of $G^{\prime}$. So both vertices $x$ and $y$ are adjacent to a vertex in $V(G) \backslash V\left(G^{\prime}\right)$. This proves the claim. Let $q=\left|V\left(G^{\prime}\right)\right|$. By Corollary 2.6 $G$ has a dominating set of size at most $(n-q) / 4+q / 3$, this is one way to dominate $G$. Since every non-outerplanar block of $G^{\prime}$ of order 6 has a vertex, that dominates all internal vertices of this block, we can choose a vertex in each block of order 6 to dominate internal vertices of $G^{\prime}$. External vertices of $G^{\prime}$ are, according to the claim above, dominated by vertices in $V(G) \backslash V\left(G^{\prime}\right)$. So we construct a dominating set by choosing all vertices in $V(G) \backslash V\left(G^{\prime}\right)$ and a vertex in each block of order 6 to dominate internal vertices of $G^{\prime}$. Since any two distinct blocks share at most one vertex, it follows that at most $\left|V\left(G^{\prime}\right)\right| / 5$ vertices have been chosen in blocks of order 6. This construction gives a dominating set of size at most $n-q+q / 5$. For all $q$ we have $\min \{(n-q) / 4+q / 3, n-q+q / 5\} \leq 17 n / 53$. 


\section{Operations on weak near-triangulations}

We start with definitions of a problematic and a bad vertex of a plane graph.

Definition 3.1 Let $G$ be a plane graph and $u \in V(G)$. We say that $u$ is a problematic vertex in $G$ if $G-u$ is not a weak near-triangulation.

Definition 3.2 Let $G$ be a plane graph and $u \in V(G)$ a problematic vertex in $G$. We say that $x$ is a $u$-bad vertex in $G$ if $x$ is not contained in a triangle of $G-u$.

If $G$ is a weak near-triangulation, then every $u$-bad vertex in $G$ is an external vertex of $G$, and it is adjacent to $u$. Moreover, if $x$ is a $u$-bad vertex, then there exists a facial triangle $u x y$ in $G$. See Fig, 1, where $u$ is a problematic vertex in $G$, and there are two $u$-bad vertices in $G$ (the white vertices). We skip the proof of the following lemma, it is similar to the proof of Lemma 2.4.

Lemma 3.3 Let $G$ be a weak near-triangulation and $x$ an external vertex of $G$. Every bounded face of $G-x$ is triangular. Moreover, if $y$ is adjacent to $x$ in $G$, then $y$ is an external vertex of $G-x$.

Lemma 3.3 has an immediate corollary.

Corollary 3.4 Let $G$ be weak near-triangulation, $D \subseteq V(G)$ a set of vertices such that $D \subseteq N[y]$ for some $y \in D$. If $D$ contains at least one external vertex of $G$, then every bounded face of $G-D$ is triangular.

Lemma 3.5 Let $G$ be a weak near-triangulation and $u$ a problematic external vertex in $G$. Let $T$ be the set of all u-bad verteces in $G$. Then $G-(\{u\} \cup T)$ is a weak near-triangulation.

Proof. Since every $u$-bad vertex in $G$ is adjacent to $u$, we find (by applying Corollary 3.4) that every face of $G-(\{u\} \cup T)$ is triangular, except possibly the unbounded face. When removing vertices that are not contained in a triangle of $G-u$ we obtain a graph in which every vertex is contained in a triangle (note that this is possibly an empty graph).

If $u$ is an internal vertex, then the following lemma applies.

Lemma 3.6 Let $G$ be a weak near-triangulation and $u$ a problematic internal vertex in $G$. Let $T$ be the set of all $u$-bad verteces in $G$, and suppose that $T \neq \emptyset$. Then $G-(\{u\} \cup T)$ is a weak near-triangulation.

Proof. We note that every $u$-bad vertex in $G$ is an external vertex of $G$. The rest of the proof is the same as the proof of Lemma 3.5 . 
Lemma 3.7 Let $G$ be a weak near-triangulation and $D \subseteq X \subseteq V(G)$ such that

(i) $G-X$ is a weak near-triangulation

(ii) $D \subseteq N[y]$ for some $y \in D$

(iii) $D$ contains at least one external vertex of $G$

then $G-D$ is a weak near-tirangulation if and only if every vertex of $X \backslash D$ is contained in a triangle of $G-D$.

Proof. If a vertex of $X \backslash D$ is not contained in a triangle of $G-D$, then $G-D$ is not a WNT.

Suppose that every vertex in $X \backslash D$ is contained in a triangle of $G-D$. By (i) $G-X$ is a WNT, so every vertex of $G-X$ is contained in a triangle of $G-X$ and hence also in a triangle of $G-D$. It follows that every vertex of $G-D$ is contained in a triangle of $G-D$. We also see that from (ii) and (iii) together with Corollary 3.4 follows that every face of $G-D$ is triangular, except possibly the unbounded face.

Lemma 3.8 Let $G$ be a weak near-triangulation, and $X \subseteq V(G)$ a set such that $G-X$ is a weak near-triangulation. Suppose that uvw is a triangle of $G$, and let $Y$ be the set of vertices of $G$ contained in the interior of uvw. Suppose that $X \cap Y=\emptyset, u \in X$ and $v, w \notin X$. If $y \in Y$ is a problematic vertex in $G-X$, then every $y$-bad vertex in $G-X$ is adjacent to $u$ in $G$.

Proof. Let $G, X, Y, y$ and $u, v, w$ be as declared in the lemma. Let $x$ be a $y$-bad vertex in $G-X$. If $x=v$ or $x=w$ there is nothing to prove, because $u v w$ is a triangle in $G$. Assume that $x \notin\{v, w\}$, and note that $x$ is adjacent to $y$, and therefore lies in the interior of $u v w$ in $G$. Since $x$ is a $y$-bad vertex in $G-X$, we find that there is a facial triangle $x y t$ in $G-X$. The edge $x t$ is an internal edge of $G$ and so there is a facial triangle $x t y^{\prime}$ in $G$, such that $y^{\prime} \neq y$. However, the triangle $x t y^{\prime}$ is not a triangle in $G-X$, for otherwise $x$ is not a $y$-bad vertex in $G-X$. It follows that $y^{\prime}$ is not in $G-X$, and therefore $y^{\prime}=u$ (because $y^{\prime}$ is adjacent to $x$ which is an internal vertex of $u v w)$.

\section{Reducibility of weak near-triangulations}

In this section we prove Theorem 2.8, Let $G$ be a weak near-triangulation and $B$ a block of $G$ that is not outerplanar. Suppose also that $B$ is not a block on 6 vertices. Let $u$ be an internal vertex of $B$ so that $u$ has at least two external neighbors (neighbors incident to the unbounded face of $G$ ). Let $u_{1}, \ldots, u_{n}$ be the external neighbors of $u$, and let $R_{1}, \ldots, R_{n}$ be regions in the plane where the region $R_{k}$ is bounded by the outer

cycle of $B$ and the edges $u u_{k}$ and $u u_{k+1}$ (indices are calculated modulo $n$ ), if $n>2$ two consecutive regions share an edge and if $n=2$ they share two edges. We may choose $u$ 
so that at most one of the regions $R_{k}, k \leq n$ contains an internal vertex of $B$ different from $u$. We note that this choice of $u$ only becomes relevant in subsection 4.4 .

The proof is divided into four main cases (depending on the number of $u$-bad vertices in $G$ ) and several subcases. For each main case there is a subsection. In the proof we work with weak near-triangulation $G$, and with subgraphs of $G$. When we say "adjacent" we mean "adjacent in $G$ " (as opposed to "adjacent in a subgraph of $G$ "), and unless otherwise stated, a facial triangle means a facial triangle in $G$.

\subsection{There are at least three $u$-bad vertices in $G$}

Suppose that $u$ has at least three $u$-bad vertices in $G$. Let $T$ be the set of all $u$-bad vertices in $G$, and define $D=\{u\} \cup T$. By Lemma 3.6, $G-D$ is a WNT. Since $|D| \geq 4$ and $D \subseteq N[u], G$ is reducible.

\subsection{There are exactly two $u$-bad vertices in $G$}

Suppose that $u$ has exactly two $u$-bad vertices in $G$, and let $x_{1}, x_{2}$ be $u$-bad vertices in $G$. By Lemma 3.6, $G-\left\{u, x_{1}, x_{2}\right\}$ is a WNT. Observe that $u$ is an internal vertex of $G$, so $u x_{1}$ and $u x_{2}$ are internal edges of $G$, and therefore there are two facial triangles $u x_{1} w_{1}$ and $u x_{1} z_{1}$ containing edge $u x_{1}$, and two facial triangles $u x_{2} w_{2}$ and $u x_{2} z_{2}$ containing edge $u x_{2}$ (note that vertices $w_{1}, x_{1}, z_{1}$ are not necessarily distinct from $w_{2}, x_{2}, z_{2}$ ). Since $x_{1}$ and $x_{2}$ are $u$-bad vertices in $G$ neither $x_{1}$ nor $x_{2}$ is contained in a triangle of $G-u$. It follows that $x_{1} w_{1}, x_{1} z_{1}$ and $x_{2} w_{2}, x_{2} z_{2}$ are external edges of $G$, and therefore $w_{1}, w_{2}, x_{1}, x_{2}$ are external vertices of $G$. Note also that if $a \neq u$ is a common neighbor of $x_{1}$ and $x_{2}$ in $G$, then $a \in\left\{w_{1}, w_{2}, z_{1}, z_{2}\right\}$, because the facial triangles containing edges $a x_{2}$ and $a x_{1}$ also contain $u$ (because $x_{1}$ and $x_{2}$ are $u$-bad vertices in $G$ ). We distinguish three possibilities. 1. $x_{1}$ and $x_{2}$ have a common neighbor different from $u$. 2. $x_{1}$ and $x_{2}$ are adjacent, and $u$ is their only common neighbor. 3. $x_{1}$ and $x_{2}$ are not adjacent, and $u$ is their only common neighbor.

\subsection{1 $x_{1}$ and $x_{2}$ have a common neighbor different from $u$}

Suppose that $x_{1}$ and $x_{2}$ have a common neighbor $t$ different from $u$. Observe that $t$ is adjacent to $u$, because $t \in\left\{w_{1}, w_{2}, z_{1}, z_{2}\right\}$. If $t$ is not problematic in $G-\left\{u, x_{1}, x_{2}\right\}$, then let $D=\left\{u, x_{1}, x_{2}, t\right\}$. Since $G-D$ is a WNT and $D \subseteq N[t], G$ is reducible. If $t$ is problematic in $G-\left\{u, x_{1}, x_{2}\right\}$, then let $T$ be the set of all $t$-bad vertices in $G-\left\{u, x_{1}, x_{2}\right\}$, and define $D=\left\{u, x_{1}, x_{2}, t\right\} \cup T$. By Lemma 3.5, $G-D$ is a WNT. Since $D \subseteq N[t], G$ is reducible.

\subsection{2 $x_{1}$ and $x_{2}$ are adjacent, and $u$ is their only common neighbor}

Observe that $u x_{1} x_{2}$ is a facial triangle in this case. Let $u x_{1} w_{1}$ and $u x_{2} w_{2}$ be facial triangles such that $w_{1} \neq x_{2}$ and $w_{2} \neq x_{1}$. Since $u$ is the only common neighbor of $x_{1}$ and $x_{2}$ we have $w_{1} \neq w_{2}$. If $w_{1}$ is not problematic in $G-\left\{u, x_{1}, x_{2}\right\}$, then define $D=\left\{u, x_{1}, x_{2}, w_{1}\right\}$. By the definition (of a problematic vertex) $G-D$ is a WNT. Since 
$|D| \geq 4$ and $D \subseteq N[u], G$ is reducible. If $w_{2}$ is not problematic in $G-\left\{u, x_{1}, x_{2}\right\}$, the reduction is analogous as above, so assume that both $w_{1}$ and $w_{2}$ are problematic in $G-\left\{u, x_{1}, x_{2}\right\}$.

Suppose that $w_{1}$ is a $w_{2}$-bad vertex in $G-\left\{u, x_{1}, x_{2}\right\}$, and that $w_{2}$ is a $w_{1}$-bad vertex in $G-\left\{u, x_{1}, x_{2}\right\}$. In this case $w_{1}$ and $w_{2}$ are adjacent, moreover $x_{1}$ and $x_{2}$ are in the exterior of triangle $w_{1} w_{2} u$, because $x_{1}$ and $x_{2}$ are external vertices of $G$. If $w_{1} w_{2} x$ is in a facial triangle and $x \neq u$, then $x$ lies either in the interior of triangle $w_{1} w_{2} u$ or in the exterior. If $x$ is in the interior of $w_{1} w_{2} u$, then the edge $x w_{1}$ is incident to a triangular face $x w_{1} t$. If $t \notin\left\{u, w_{2}\right\}$, then $x w_{1} t$ is a triangle in $G-\left\{u, x_{1}, x_{2}, w_{2}\right\}$, and so $w_{1}$ is not a $w_{2}$-bad vertex in $G-\left\{u, x_{1}, x_{2}\right\}$, contrary to the assumption. It follows that $x w_{1} u$ and $x w_{1} w_{2}$ are facial triangles in $G$. Similarly, since $w_{2}$ is a $w_{1}$-bad vertex in $G-\left\{u, x_{1}, x_{2}\right\}$, we find that $x w_{2} u$ is a facial triangle in $G$. This in particular implies that $x$ is the only vertex of $G$ in the interior of triangle $w_{1} w_{2} u$.

(a)

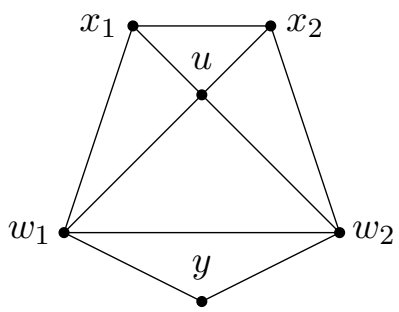

(c)

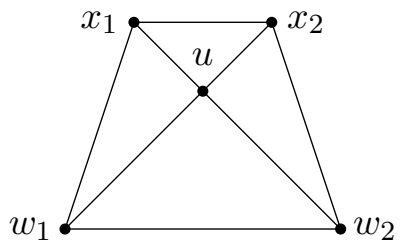

(b)

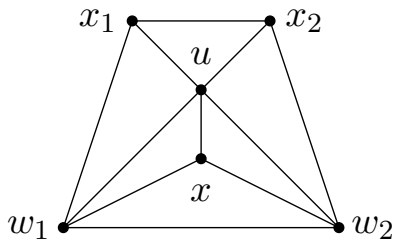

$(d)$

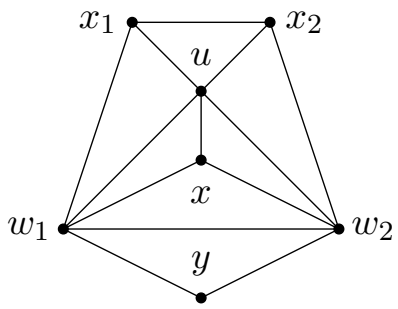

Figure 2: Cases $(a)$ to $(d)$.

Suppose now that $w_{1} w_{2} y$ is a facial triangle such that $y$ is in the exterior of $w_{1} w_{2} u$. Since $w_{1}, w_{2}, x_{1}, x_{2}$ are external vertices of $G, y \neq x_{1}$ and $y \neq x_{2}$. If $y w_{1}$ is not an external edge of $G$, then there is a facial triangle $y w_{1} t$, with $t \neq x_{1}, x_{2}$ (note again that $w_{1}, w_{2}, x_{1}, x_{2}$ are external vertices of $G$ ). This contradicts the assumption that $w_{1}$ is a $w_{2}$-bad vertex in $G-\left\{u, x_{1}, x_{2}\right\}$. Therefore $w_{1} y$ is an external edge of $G$, and similarly $w_{2} y$ is an external edge of $G$. Since $y w_{1}, y w_{2}, w_{1} x_{1}, x_{1} x_{2}$ and $x_{2} w_{2}$ are external edges of $G$, and also $w_{1} w_{2}$ is an external edge if there is no facial triangle $w_{1} w_{2} y$ (with $y$ in the exterior of $w_{1} w_{2} u$ ), we find that $B$ is isomorphic to one of the four graphs shown in Fig. 2, $B$ is a block on 6 vertices in cases (a) and (b), which contradicts our assumptions. 
$B$ is a block on 5 vertices in case (c), and since $G-\left\{u, x_{1}, x_{2}\right\}$ is a WNT, $w_{1}$ is contained in a facial triangle $w_{1} a b$ of $G-\left\{u, x_{1}, x_{2}\right\}$. Clearly, $a, b \notin B$, and therefore $w_{1}$ is not a $w_{2}$-bad vertex in $G-\left\{u, x_{1}, x_{2}\right\}$ (contrary to the assumption). In case (d), $B$ is a block of order 7. If there is a $w_{1}$-bad vertex $z \notin\left\{x, w_{2}\right\}$ in $G-\left\{u, x_{1}, x_{2}\right\}$, then let $T$ be the set of all $w_{1}$-bad vertices in $G-\left\{u, x_{1}, x_{2}\right\}$, and define $D=\left(\left\{w_{1}, x_{1}\right\} \cup T\right) \backslash\left\{w_{2}\right\}$. Note that $x$ is a $w_{1}$-bad vertex in $G-\left\{u, x_{1}, x_{2}\right\}$ and so $x, z, x_{1}, w_{1} \in D$. It follows that $|D| \geq 4$, moreover $D \subseteq N\left[w_{1}\right]$. Since $G-\left\{u, x_{1}, x_{2}\right\}$ is a WNT, Lemma 3.5] implies that $G-\left(\left\{u, x_{1}, x_{2}, w_{1}\right\} \cup T\right)$ is a WNT. Since $\left(\left\{u, x_{1}, x_{2}, w_{1}\right\} \cup T\right) \backslash D=\left\{u, x_{2}, w_{2}\right\}$ we find, by applying Lemma 3.7, that $G-D$ is a WNT, and therefore $G$ is reducible. (When applying Lemma 3.7 we set $X=\left\{u, x_{1}, x_{2}, w_{1}\right\} \cup T$, and we note that $X-D$ induces a triangle.) If $x$ and $w_{2}$ are the only $w_{1}$-bad vertices in $G-\left\{u, x_{1}, x_{2}\right\}$, then define $D=\left\{u, x_{1}, x_{2}, w_{1}, w_{2}, x\right\}$. Apply Lemma 3.5 to $G-\left\{u, x_{1}, x_{2}\right\}$ (recall that $G-\left\{u, x_{1}, x_{2}\right\}$ is a WNT), and its problematic vertex $w_{1}$. It follows that $G-D$ is a WNT, because $x$ and $w_{2}$ are the only $w_{1}$-bad vertices in $G-\left\{u, x_{1}, x_{2}\right\}$. Reducibility of $G$ follows from $D \subseteq N[u]$. This proves that $w_{1}$ is not a $w_{2}$-bad vertex, or $w_{2}$ is not a $w_{1}$-bad vertex in $G-\left\{u, x_{1}, x_{2}\right\}$.

Assume, without loss of generality, that $w_{2}$ is not a $w_{1}$-bad vertex in $G-\left\{u, x_{1}, x_{2}\right\}$. Let $T$ be the set of all $w_{1}$-bad vertices in $G-\left\{u, x_{1}, x_{2}\right\}$, and suppose first that $|T| \geq 2$. Define $D=\left\{x_{1}, w_{1}\right\} \cup T$. By Lemma 3.5. $G-\left(\left\{u, x_{1}, x_{2}, w_{1}\right\} \cup T\right)$ is a WNT, and therefore by Lemma 3.7, $G-D$ is a WNT if and only if $u$ and $x_{2}$ are contained in a triangle of $G-D$. Since $u x_{2} w_{2}$ is a triangle in $G-D$, we conclude that $G-D$ is a WNT. Moreover $D \subseteq N\left[w_{1}\right]$ and $|D| \geq 4$, therefore $G$ is reducible. Suppose now that there is exactly one $w_{1}$-bad vertex in $G-\left\{u, x_{1}, x_{2}\right\}$. Call it $z$, and note that $z \neq w_{2}$, and that $G-\left\{u, x_{1}, x_{2}, w_{1}, z\right\}$ is a WNT by Lemma 3.5.

The edge $w_{1} z$ is incident to a facial triangle in $G$ (and in $G-\left\{u, x_{1}, x_{2}\right\}$ ). Suppose that there exists a vertex $t \neq w_{2}$ such that $w_{1} z t$ is a facial triangle. If $t$ is not problematic in $G-\left\{u, x_{1}, x_{2}, w_{1}, z\right\}$ then let $D=\left\{x_{1}, w_{1}, z, t\right\}$. Since $G-\left\{u, x_{1}, x_{2}, w_{1}, z, t\right\}$ is a WNT, and vertices $u$ and $x_{2}$ are contained in the triangle $u x_{2} w_{2}$ of $G-D$, we find by Lemma 3.7, that $G-D$ is a WNT. So $G$ is reducible, because $D \subseteq N\left[w_{1}\right]$. If $t$ is problematic in $G-\left\{u, x_{1}, x_{2}, w_{1}, z\right\}$, then let $T$ be the set of all $t$-bad vertices in $G$ $\left\{u, x_{1}, x_{2}, w_{1}, z\right\}$. Define $D=\left\{w_{1}, z, t\right\} \cup T$. By Lemma 3.5, $G-\left\{u, x_{1}, x_{2}, w_{1}, z, t\right\} \cup T$ is a WNT, and so $G-D$ is a WNT by Lemma 3.7, due to the fact that vertices $u, x_{1}, x_{2}$ are contained in a triangle of $G-D$. Since $D \subseteq N[t]$ and $|D| \geq 4$, we find that $G$ is reducible. In both cases we found that $G$ is reducible, so assume that the only facial triangle incident to $w_{1} z$ is the triangle $w_{1} z w_{2}$. We discuss two possibilities.

First is when $z$ is in the interior of the triangle $w_{1} w_{2} u$. Then $w_{1} z$ is an internal edge of $G$, so it's contained in two facial triangles. If one of them is $w_{1} z u$, then $z$ is adjacent to $u$ in $G$. In this case define $D=\left\{u, x_{1}, x_{2}, w_{1}, z\right\}$ and observe that $G-D$ is a WNT, and that $D \subseteq N[u]$, hence $G$ is reducible. Otherwise, if $w_{1} z u$ is not a facial triangle, there exists a vertex $t \neq w_{2}$ such that $w_{1} z t$ is a facial triangle, contradicting the assumption that $w_{1} z w_{2}$ is the only facial triangle containing the edge $w_{1} z$.

The second possibility is that $z$ is in the exterior of triangle $w_{1} w_{2} u$. Since $w_{1} z w_{2}$ is the only facial triangle incident to $w_{1} z$ we find that $w_{1} z$ is an external edge of $G$. Similarly, since $z$ is a $w_{1}$-bad vertex in $G-\left\{u, x_{1}, x_{2}\right\}$, it is not contained in a triangle 
of $G-\left\{u, x_{1}, x_{2}, w_{1}\right\}$, and so $z w_{2}$ is an external edge of $G$. It follows that edges $w_{2} x_{2}, x_{2} x_{1}, x_{1} w_{1}, w_{1} z, z w_{2}$ are external edges of $G$, and so the union of these edges is the boundary of $B$. If there is no vertex in the interior of the triangle $w_{1} w_{2} u$, then $B$ is a block of order 6 . Otherwise $u$ is adjacent to a vertex $y$ in the interior of $w_{1} w_{2} u$. If $y$ is not problematic in $G-\left\{u, x_{1}, x_{2}\right\}$, then let $D=\left\{u, x_{1}, x_{2}, y\right\} . G-D$ is a WNT, by the definition of a problematic vertex, and since $D \subseteq N[u], G$ is reducible. Assume therefore that $y$ is problematic in $G-\left\{u, x_{1}, x_{2}\right\}$. We apply Lemma 3.8, where $X=\left\{u, x_{1}, x_{2}\right\}$ and where the triangle $u w_{1} w_{2}$ takes the role of $u v w$ in Lemma 3.8. The lemma implies that every $y$-bad vertex in $G-\left\{u, x_{1}, x_{2}\right\}$ is adjacent to $u$ in $G$. Let $T$ be the set of all $y$-bad vertices in $G-\left\{u, x_{1}, x_{2}\right\}$. Define $D=\left\{u, x_{1}, x_{2}, y\right\} \cup T$, and note that $D \subseteq N[u]$. By Lemma 3.5, $G-D$ is a WNT, therefore $G$ is reducible.

\subsection{3 $x_{1}$ and $x_{2}$ are not adjacent, and $u$ is their only common neighbor}

Let $w_{1}, z_{1}, w_{2}, z_{2}$ be pairwise distinct vertices such that $u x_{1} w_{1}$ and $u x_{1} z_{1}$ are facial triangles containing $u x_{1}$, and $u x_{2} w_{2}$ and $u x_{2} z_{2}$ are facial triangles containing $u x_{2}$. Note that $x_{1}$ and $x_{2}$ are external vertices of $G$, and therefore $w_{1}$ is not adjacent to $z_{1}$. Moreover, there is no path in $G$ that avoids $x_{1}, x_{2}$ and $u$ between $w_{1}$ and $z_{1}$ (again, because $x_{1}$ and $x_{2}$ are external vertices). Similarly there is no path in $G$ that avoids $x_{1}, x_{2}$ and $u$ between $w_{1}$ and $w_{2}$, or there is no path in $G$ that avoids $x_{1}, x_{2}$ and $u$ between $w_{1}$ and $z_{2}$ (if both paths exist, then $x_{1}$ or $x_{2}$ is not an external vertex). Assume, without loss of generality, the latter. This, in particular, implies that $w_{1}$ is not adjacent to $z_{2}$ in $G$.

If $w_{1}$ is not problematic in $G-\left\{u, x_{1}, x_{2}\right\}$, then we define $D=\left\{u, x_{1}, x_{2}, w_{1}\right\}$. Since $D \subseteq N[u]$ and $G-D$ is a WNT, $G$ is reducible. Assume therefore that $w_{1}$ is problematic in $G-\left\{u, x_{1}, x_{2}\right\}$. If $w_{1}$ has at least two $w_{1}$-bad vertices in $G-\left\{u, x_{1}, x_{2}\right\}$ then let $T$ be the set of $w_{1}$-bad vertices in $G-\left\{u, x_{1}, x_{2}\right\}$ and define $D=\left\{w_{1}, x_{1}\right\} \cup T$. Since $w_{1}$ is not adjacent to $z_{2}$, we find that $z_{2} \notin T$. It follows that $u x_{2} z_{2}$ is a triangle of $G-D$. We apply Lemma 3.7 to sets $X=\left\{u, x_{1}, x_{2}, w_{1}\right\} \cup T$ and $D$. Since $G-X$ is a WNT, and every vertex of $X \backslash D$ is contained in a triangle of $G-D$, we find that $G-D$ is a WNT. Since $D \subseteq N\left[w_{1}\right]$ and $|D| \geq 4, G$ is reducible. Assume now that there is exactly one $w_{1}$-bad vertex in $G-\left\{u, x_{1}, x_{2}\right\}$, call this vertex $z$. Let $w_{1} z t$ be a facial triangle. If $t$ is not problematic in $G-\left\{u, x_{1}, x_{2}, w_{1}, z\right\}$, then let $D=\left\{x_{1}, w_{1}, z, t\right\}$. Since $z_{2}$ is not adjacent to $w_{1}$, we find that both $z$ and $t$ are distinct from $z_{2}$. It follows that $u$ and $x_{2}$ are contained in a triangle of $G-D$, the triangle $u x_{2} z_{2}$. By Lemma 3.7. $G-D$ is a WNT, moreover $D \subseteq N\left[w_{1}\right]$. Therefore $G$ is reducible. The last possibility is that $t$ is problematic in $G-\left\{u, x_{1}, x_{2}, w_{1}, z\right\}$. In this case let $T$ be the set of all $t$-bad vertices in $G-\left\{u, x_{1}, x_{2}, w_{1}, z\right\}$, and define $D=\left\{t, z, w_{1}\right\} \cup T$. Since there is no path between $w_{1}$ and $z_{2}$ in $G$ that avoids $x_{1}, x_{2}$ and $u$, we find that $z_{2} \notin T \cup\{z, t\}$. Analogous arguments prove that $z_{1} \notin T \cup\{z, t\}$. It follows that vertices $u, x_{1}$, and $x_{2}$ are contained in triangles of $G-D$, these are triangles $u x_{1} z_{1}$ and $u x_{2} z_{2}$. By Lemma 3.5. $G-\left(\left\{u, x_{1}, x_{2}, w_{1}, z, t\right\} \cup T\right)$ is a WNT. We apply Lemma 3.7 to find that $G-D$ is a WNT. Moreover $D \subseteq N[t]$, so $G$ is reducible. 


\subsection{There is exactly one $u$-bad vertex in $G$}

Suppose that there is exactly one $u$-bad vertex in $G$, call it $x_{1}$. By Lemma 3.6, $G-$ $\left\{u, x_{1}\right\}$ is a WNT. Let $u x_{1} w_{1}$ and $u x_{1} z_{1}$ be facial triangles containing the edge $u x_{1}$. Note that $x_{1} z_{1}$ and $x_{1} w_{1}$ are external edges of $G$, and therefore $w_{1}$ and $z_{1}$ are external vertices of $G$. If $w_{1}$ is problematic in $G-\left\{u, x_{1}\right\}$, then let $T$ be the set of all $w_{1}$-bad vertices in $G-\left\{u, x_{1}\right\}$. Define $D=\left\{u, x_{1}, w_{1}\right\} \cup T$. By Lemma 3.5, $G-D$ is a WNT. Since $D \subseteq N\left[w_{1}\right], G$ is reducible.

Suppose that $w_{1}$ is not problematic in $G-\left\{u, x_{1}\right\}$, and suppose additionaly that $z_{1}$ is not problematic in $G-\left\{u, x_{1}, w_{1}\right\}$. Then define $D=\left\{u, x_{1}, w_{1}, z_{1}\right\}$, and observe that $G-D$ is a WNT. Since $D \subseteq N[u], G$ is a reducible.

It remains to prove that $G$ is reducible if $z_{1}$ is problematic in $G-\left\{u, x_{1}, w_{1}\right\}$. If $z_{1}$ is adjacent to $w_{1}$ in $G$, then let $T$ be the set of all $z_{1}$-bad vertices in $G-\left\{u, x_{1}, w_{1}\right\}$. Define $D=\left\{u, w_{1}, x_{1}, z_{1}\right\} \cup T$ and observe that $D \subseteq N\left[z_{1}\right]$. By Lemma 3.5, $G-D$ is a WNT, and so $G$ is reducible. From now on we assume that $w_{1}$ and $z_{1}$ are not adjacent in $G$.

If $\operatorname{deg}_{B}\left(w_{1}\right)=3$, then $w_{1}$ is contained in a triangle of $G-\left\{u, x_{1}\right\}$, say $w_{1} a b$, such that $a, b \notin B$. In this case let $T$ be the set of all $z_{1}$-bad vertices in $G-\left\{u, x_{1}, w_{1}\right\}$, and define $D=\left\{u, x_{1}, z_{1}\right\} \cup T$. Since $G-\left(\left\{u, x_{1}, w_{1}, z_{1}\right\} \cup T\right)$ is a WNT, also $G-D$ is a WNT by Lemma 3.7 (note that $a, b \notin T$, and so $w_{1}$ is contained in a triangle of $G-D)$. Since $D \subseteq N\left[z_{1}\right]$, we find that $G$ is reducible. Therefore $\operatorname{deg}_{B}\left(w_{1}\right) \geq 4$.

Now we can prove the following claim: every $z_{1}$-bad vertex in $G-\left\{u, x_{1}, w_{1}\right\}$ is adjacent to $w_{1}$ in $G$. If not, then the set of $z_{1}$-bad vertices in $G-\left\{u, x_{1}, w_{1}\right\}$ that are not adjacent to $w_{1}$ in $G$ is nonempty, call this set $T$. Also, let $X$ be the set that contains $u, x_{1}, w_{1}, z_{1}$ and all $z_{1}$-bad vertices in $G-\left\{u, x_{1}, w_{1}\right\}$. By Lemma 3.5, $G-X$ is a WNT. Define $D=\left\{u, x_{1}, z_{1}\right\} \cup T$, and observe that $G-D$ contains all neighbors of $w_{1}$ in $G$, except $x_{1}$ and $u$. Moreover, every vertex (distinct from $w_{1}$ ) in $X \backslash D$ is adjacent to $w_{1}$ in $G-D$. Since $\operatorname{deg}_{B}\left(w_{1}\right) \geq 4$, and $w_{1} x_{1}$ is an external edge of $B$, and $u x_{1} w_{1}$ is a facial triangle, we find that every vertex in $X \backslash D$ is contained in a triangle of $G-D$. Hence, by Lemma 3.7, $G-D$ is a WNT. Since $D \subseteq N\left[z_{1}\right], G$ is reducible. This proves the claim.

If every $z_{1}$-bad vertex in $G-\left\{u, x_{1}, w_{1}\right\}$ is adjacent to $u$, then let $T$ be the set of all $z_{1}$-bad vertices in $G-\left\{u, x_{1}, w_{1}\right\}$. Define $D=\left\{u, x_{1}, w_{1}, z_{1}\right\} \cup T$. Since $D \subseteq N[u]$, and $G-D$ is a WNT, $G$ is reducible. Assume therefore that a $z_{1}$-bad vertex is not adjacent to $u$.

Let $y$ be a $z_{1}$-bad vertex in $G-\left\{u, x_{1}, w_{1}\right\}$ not adjacent to $u$ in $G$. Since $w_{1} z_{1} \notin E(G)$ and $y u \notin E(G)$ there exist exactly one vertex adjacent to $y$ in the interior of 4-cycle $y w_{1} u z_{1}$ (for otherwise $y$ is not a $z_{1}$-bad vertex in $G-\left\{u, x_{1}, w_{1}\right\}$ ). Call this vertex $z$ and observe that $z y w_{1}$ and $z y z_{1}$ are facial triangles. If $z$ and $u$ are not adjacent then let $w_{1} z y_{1}$ be the facial triangle such that $y_{1} \neq y$. Let $T$ be the set of all $z_{1}$-bad vertices in $G-\left\{u, w_{1}, x_{1}\right\}$, and define $D=\left(\left\{u, x_{1}, z_{1}\right\} \cup T\right) \backslash\left\{z, y_{1}\right\}$. Since $G-\left\{u, w_{1}, x_{1}, z_{1}\right\} \cup T$ is a WNT, also $G-D$ is a WNT (by Lemma 3.7). As $y \in D$, we have $|D| \geq 4$ and so $G$ is reducible. It follows that $z$ and $u$ are adjacent. Moreover the triangle $w_{1} u z$ is a facial triangle (we do the same reduction as above if $w_{1} u z$ is not a facial triangle). 
Suppose that there is a vertex $x$ in the interior of triangle $u z z_{1}$, such that $x$ is adjacent to $u$ in $G$. If $x$ is not problematic in $G-\left\{u, x_{1}, w_{1}\right\}$, then let $D=\left\{u, x_{1}, w_{1}, x\right\}$ and observe that $G-D$ is a WNT. Since $D \subseteq N[u], G$ is reducible. Suppose that $x$ is problematic in $G-\left\{u, x_{1}, w_{1}\right\}$. By Lemma 3.8, every $x$-bad vertex in $G-\left\{u, x_{1}, w_{1}\right\}$ is adjacent to $u$. Let $T$ be the set of all $x$-bad vertices in $G-\left\{u, x_{1}, w_{1}\right\}$. Define $D=\left\{u, x_{1}, w_{1}, x\right\} \cup T$ and observe that $D \subseteq N[u]$. By Lemma 3.5, $G-D$ is a WNT, and therefore $G$ is reducible. It follows that $u z_{1} z$ is a facial triangle of $G$, and therefore $z$ is also a $z_{1}$-bad vertex in $G-\left\{u, x_{1}, w_{1}\right\}$.

If the set $T$ of all $z_{1}$-bad vertices in $G-\left\{u, x_{1}, w_{1}\right\}$ contains more than two vertices, then let $D=\left(\left\{u, x_{1}, z_{1}\right\} \cup T\right) \backslash\{z, y\}$. Since $z y w_{1}$ is a triangle in $G-D$ we find, by applying Lemma 3.7, that $G-D$ is a WNT. Since $D \subseteq N\left[z_{1}\right], G$ is reducible. Assume therefore that $z$ and $y$ are the only $z_{1}$-bad vertices in $G-\left\{u, x_{1}, w_{1}\right\}$, and so $G-\left\{u, x_{1}, w_{1}, z_{1}, z, y\right\}$ is a WNT. If $w_{1} y$ is not an external edge of $G$, then define $D=\left\{u, x_{1}, z_{1}, z\right\}$. Since $w_{1} y$ is not an externl edge of $G, y$ and $w_{1}$ are contained in a triangle of $G-D$. Lemma 3.7 implies that $G-D$ is a WNT. Reducibility of $G$ follows from $D \subseteq N\left[z_{1}\right]$. Assume therefore that $y w_{1}$ is an external edge of $G$. If $y z_{1}$ is not an external edge of $G$, then define $D=\left\{u, x_{1}, w_{1}, z\right\}$. Since $y$ and $z_{1}$ are contained in a triangle of $G-D$ (note that there is a facial triangle $y z_{1} t$, where $t \neq z$, and $t \neq w_{1}$ because $z_{1}$ is not adjacent to $w_{1}$, and $t \neq x_{1}$ because $z_{1}$ is an external vertex of $G$ ), we find that $G-D$ is a WNT. Reducibility follows from $D \subseteq N[u]$. Hence also $z_{1} y$ is an external edge of $G$, and therefore all four edges $w_{1} x_{1}, x_{1} z_{1}, z_{1} y$ and $y w_{1}$ are external edges of $G$. So $B$ is a block of order 6 .

\subsection{There are no $u$-bad vertices in $G$}

Suppose that $u$ has no $u$-bad vertices in $G$. Let $u_{1}$ be any external neighbor of $u$ in $G$. Since $u$ is not problematic in $G, u_{1}$ is contained in a triangle of $G-u$. Note first that $\operatorname{deg}_{B}\left(u_{1}\right)>2$, because $u_{1}$ is adjacent to $u$ in $B$, and $u$ is an internal vertex of $B$.

We claim the following: if $\operatorname{deg}_{B}\left(u_{1}\right)=3$ then $G$ is reducible or $u_{1}$ is contained in a triangle $u_{1} a b$, where $a, b \notin B$. Suppose that $\operatorname{deg}_{B}\left(u_{1}\right)=3$. Let $z_{1}, z_{2}$ and $u$ be neighbors of $u_{1}$ in $B$, where $z_{1}$ and $z_{2}$ are external vertices of $B$. Since $u_{1}$ is contained in a triangle of $G-u$ we find that $u_{1}$ is contained in a triangle $u_{1} a b$, where $a, b \notin B$, or $z_{1}$ is adjacent to $z_{2}$. Suppose that $z_{1}$ is adjacent to $z_{2}$ and that $u_{1}$ is not contained in a triangle $u_{1} a b$, where $a, b \notin B$. Then every block $B^{\prime} \neq B$ of $G$ containing $u_{1}$ is a $K_{2}$, and therefore $u_{1} z_{1} z_{2}$ is the only triangle containing $u_{1}$ in $G-u$. It follows that $z_{1}$ and $z_{2}$ are the only vertices that are potentially not in a triangle of $G-\left\{u, u_{1}\right\}$.

Suppose that $\operatorname{deg}(u) \geq 4$ or $z_{1} z_{2}$ is an internal edge of $G$. Then all vertices of $G-\left\{u, u_{1}\right\}$ are contained in a triangle of $G-\left\{u, u_{1}\right\}$, and so (note that by Corollary 3.4 all bounded faces of $G-\left\{u, u_{1}\right\}$ are triangular), $G-\left\{u, u_{1}\right\}$ is a WNT. In this case the reductions are defined as follows. If $z_{1}$ is problematic in $G-\left\{u, u_{1}\right\}$, then let $T$ be the set of all $z_{1}$-bad vertices in $G-\left\{u, u_{1}\right\}$, and define $D=\left\{u, u_{1}, z_{1}\right\} \cup T$. Since $G-D$ is a WNT and $D \subseteq N\left[z_{1}\right], G$ is reducible. If $z_{1}$ is not problematic in $G-\left\{u, u_{1}\right\}$ and $z_{2}$ is not problematic in $G-\left\{u, u_{1}, z_{1}\right\}$, then let $D=\left\{u, u_{1}, z_{1}, z_{2}\right\}$. Since $D \subseteq N[u], G$ is reducible. If $z_{2}$ is problematic in $G-\left\{u, u_{1}, z_{1}\right\}$, then let $T$ be the set of all $z_{2}$-bad 
vertices in $G-\left\{u, u_{1}, z_{1}\right\}$. Define $D=\left\{u, u_{1}, z_{1}, z_{2}\right\} \cup T$. Since $z_{1}$ is adjacent to $z_{2}$, $D \subseteq N\left[z_{2}\right]$. Since $G-D$ is a WNT, $G$ is reducible.

It remains to define reductions when $z_{1} z_{2}$ is an external edge of $G$ and $\operatorname{deg}(u)=3$. In this case $B$ is a $K_{4}$ (recall that $\operatorname{deg}_{B}\left(u_{1}\right)=3$, therefore $z_{1} u_{1}$ and $z_{2} u_{1}$ are external edges of $B$ ), and $G-u$ is a WNT. If there is a $z_{1}$-bad vertex $t \neq u_{1}$ in $G-u$, then let $T$ be the set of all $z_{1}$-bad vertices in $G-u$, and define $D=\left\{u, z_{1}\right\} \cup T$. Since $u_{1} \in T$ we have $|T| \geq 2$, and therefore $|D| \geq 4$. Since $G-u$ is a WNT, we find (by applying Lemma 3.5) that $G-D$ is a WNT. Reducibility follows from $D \subseteq N\left[z_{1}\right]$. Therefore $u_{1}$ is the only $z_{1}$-bad vertex in $G-u$, and similarly $u_{1}$ is also the only $z_{2}$-bad vertex in $G-u$. It follows that there exist blocks $B_{1}$ and $B_{2}$ of $G$, both different from $B$, and both near-triangulations, such that $z_{1} \in B_{1}$ and $z_{2} \in B_{2}$. So we may define $D=\left\{u, u_{1}, z_{1}, z_{2}\right\}$. Observe that $G-D$ is a WNT because none of the vertices $u_{1}, z_{1}$ and $z_{2}$ have a bad vertex in $G-u$ which is not contained in $B$. Since $D \subseteq N[u], G$ is reducible. This proves the claim, and therefore it remains to define reductions in case if $\operatorname{deg}_{B}\left(u_{1}\right) \geq 4$, or $\operatorname{deg}_{B}\left(u_{1}\right)=3$ and $u_{1}$ is contained in a triangle $u_{1} a b$, where $a, b \notin B$. Assume in the sequal that $\operatorname{deg}_{B}\left(u_{1}\right) \geq 4$, or $\operatorname{deg}_{B}\left(u_{1}\right)=3$ and $u_{1}$ is contained in a triangle $u_{1} a b$, where $a, b \notin B$, moreover assume that this is true for any external neighbor $u_{1}$ of $u$. This in particular implies that every vertex of $G-u$ is contained in a facial triangle of $G-u$ which is also a facial triangle of $G$ (or equvalently: every vertex of $G-u$ is contained in a facial triangle of $G$ not containing $u$ ).

\subsection{1 $u_{1}$ is not problematic in $G-u$}

Suppose that $u_{1}$ is not problematic in $G-u$, and therefore $G-\left\{u, u_{1}\right\}$ is a WNT. The edge $u u_{1}$ is incident to two triangular faces of $G$. So there are vertices $x$ and $y$ such that $u u_{1} x$ and $u u_{1} y$ are facial triangles in $G$. By the choice of $u$ (see Section 4), at least one of $x$ and $y$ is an external vertex of $B$ (and $G$ ), for otherwise more than one region $R_{k}, k \leq n$ contains an internal vertex of $B$. Assume that $x$ is an external vertex. If $u_{1} y$ is an external edge of $G$, then let $u_{2}=y$, otherwise let $u_{2}=x$.

We claim that $u_{1}$ is contained in a triangle of $G-\left\{u, u_{2}\right\}$. If $\operatorname{deg}_{B}\left(u_{1}\right)=3$ then $u_{1}$ is contained in a triangle $u_{1} a b$, where $a, b \notin B$ (in which case the claim is true). Assume now that $\operatorname{deg}_{B}\left(u_{1}\right) \geq 4$. Now if $u_{2}=y$ then $u_{1} u_{2}$ is an external edge of $B$, and $u u_{1} u_{2}$ is a facial triangle. Since $\operatorname{deg}_{B}\left(u_{1}\right) \geq 4$ and $B$ is a near-triangulation we find that $u_{1}$ is incident to at least three facial triangles of $B$. When we remove $u$ and $u_{1}$ from $G$ at least one facial triangle containing $u_{1}$ remains. If $u_{2}=x$ and $u_{1} x$ is an external edge, then the argument is the same as above. So assume that $u_{1} x$ is also not an external edge (we already know that $u_{1} y$ is not an external edge). Now in this case $\operatorname{deg}_{B}\left(u_{1}\right) \geq 5$. Removing vertices $u$ and $u_{2}$ (which form a facial triangle with $u_{1}$ ) keeps at least one facial triangle containing $u_{1}$. This proves the claim.

If $u_{2}$ is a problamatic vertex in $G-\left\{u, u_{1}\right\}$, then let $T$ be the set of all $u_{2}$-bad vertices in $G-\left\{u, u_{1}\right\}$. Since $G-\left\{u, u_{1}\right\}$ is a WNT, it follows from Lemma 3.5 that $G-\left(\left\{u, u_{1}, u_{2}\right\} \cup T\right)$ is a WNT. We define $D=\left\{u, u_{1}, u_{2}\right\} \cup T$. Since $D \subseteq N\left[u_{2}\right]$, $|D| \geq 4$ and $G-D$ is a WNT, we find that $G$ is reducible.

Assume that $u_{2}$ is not problematic in $G-\left\{u, u_{1}\right\}$ and so $G-\left\{u, u_{1}, u_{2}\right\}$ is a WNT. 
Let $t \neq u_{1}$ be such that $u u_{2} t$ is a facial triangle. If $t$ is not problematic in $G-\left\{u, u_{1}, u_{2}\right\}$ then let $D=\left\{u, u_{1}, u_{2}, t\right\}$. We have $D \subseteq N[u]$ and $G-D$ is a WNT, so $G$ is reducible. So assume that $t$ is problematic in $G-\left\{u, u_{1}, u_{2}\right\}$.

Let $T$ be the set of all $t$-bad vertices in $G-\left\{u, u_{1}, u_{2}\right\}$. If $t$ and $u_{1}$ are adjacent in $G$ then let $D=\left\{u, u_{1}, u_{2}, t\right\} \cup T$. We find that $G-D$ is a WNT according to Lemma 3.5. Moreover $D \subseteq N[t]$, and so $G$ is reducible. Assume therefore that $t$ is not adjacent to $u_{1}$. If no vertex of $T$ is adjacent to $u_{1}$ then let $D=\left\{u, u_{2}, t\right\} \cup T$. It follows from Lemma 3.7 and the fact that $u_{1}$ is contained in a triangle of $G-\left\{u, u_{2}\right\}$ (and so also in a triangle of $G-D$ ) that $G-D$ is a WNT. Since $D \subseteq N[t], G$ is reducible. Assume from now on that a vertex of $T$ is adjacent to $u_{1}$, and call this vertex $t_{0}$.

Case $(a)$. Suppose that $t_{0}$ is not adjacent to $u$ in $G$. Then the interior of 4 cycle $t_{0} u_{1} u t$ contains exactly one vertex adjacent to $t_{0}$, call it $z$ (if the interior of $t_{0} u_{1} u t$ contains two vertices adjacent to $t_{0}$, then $t_{0}$ is contained in a triangle of $G-\left\{u, u_{1}, u_{2}, t\right\}$, and hence $t_{0}$ is not a $t$-bad vertex in $\left.G-\left\{u, u_{1}, u_{2}\right\}\right)$. If $z$ and $u$ are not adjacent in $G$, then $u_{1}$ is contained in a triangle $u_{1} z z^{\prime}$, where $z^{\prime}$ lies in the interior of $u_{1} u t z$. Let $T$ be the set of all $t$-bad vertices in $G-\left\{u, u_{1}, u_{2}\right\}$, and define $D=\left\{u, u_{2}, t\right\} \cup T \backslash\left\{z, z^{\prime}\right\}$. Since $G-\left(\left\{u, u_{1}, u_{2}, t\right\} \cup T\right)$ is a WNT, we find (by applying Lemma 3.7) that $G-D$ is a WNT. Since $t_{0} \in D$, we have $|D| \geq 4$. Moreover $D \subseteq N[t]$, and therefore $G$ is reducible.

(a)

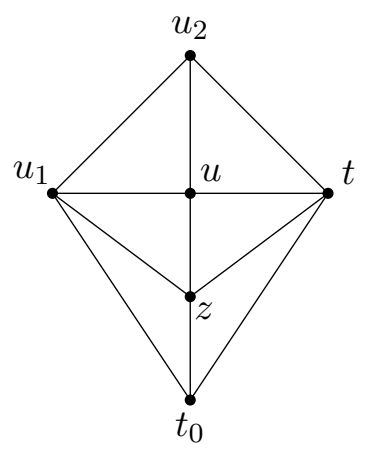

(b)

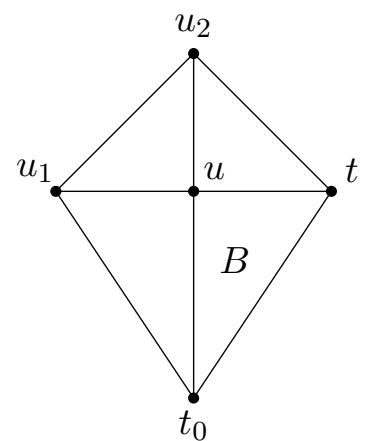

Figure 3: Case $(a): t_{0}$ is not adjacent to $u$, Case $(b): t_{0}$ is adjacent to $u$.

Assume therefore that $z$ and $u$ are adjacent in $G$ (see Fig. 3, Case $(a)$ ). If the interior of the triangle $u_{1} u z$ contains a vertex, then $u_{1}$ is contained in a triangle $u_{1} z z^{\prime}$, where $z^{\prime}$ lies in the interior of $u_{1} u z$. In this case we can do the same reduction as in the previous case, by defining $D=\left\{u, u_{2}, t\right\} \cup T \backslash\left\{z, z^{\prime}\right\}$. We may therefore assume that there is no vertex of $G$ in the interior of $u u_{1} z$.

Suppose that $y$ is a vertex of $G$ in the interior of $u t z$ adjacent to $u$. If $y$ is not problematic in $G-\left\{u, u_{1}, u_{2}\right\}$, we define $D=\left\{u, u_{1}, u_{2}, y\right\}$. Since $G-D$ is a WNT, and $D \subseteq N[u], G$ is reducible. Otherwise, if $y$ is problematic in $G-\left\{u, u_{1}, u_{2}\right\}$, then by Lemma 3.8, every $y$-bad vertex in $G-\left\{u, u_{1}, u_{2}\right\}$ is adjacent to $u$ in $G$. In this case 
let $T$ be the set of all $y$-bad vertices in $G-\left\{u, u_{1}, u_{2}\right\}$ and define $D=\left\{u, u_{1}, u_{2}, y\right\} \cup T$. Since $G-D$ is a WNT, and $D \subseteq N[u]$, we find that $G$ is reducible. This proves that there is no vertex of $G$ in the interior of $u t z$. Observe that we proved that all triangles on Fig. 3, Case $(a)$, are facial triangles.

Let $T$ be the set of all $t$-bad vertices in $G-\left\{u, u_{1}, u_{2}\right\}$, and let $X=\left\{u, u_{1}, u_{2}, t\right\} \cup T$. If $|T| \geq 3$, then $D=X \backslash\left\{u_{1}, z, t_{0}\right\}$. Since $G-X$ is a WNT, also $G-D$ is a WNT, according to Lemma 3.7. Since $D \subseteq N[t]$, and $D \geq 4, G$ is reducible. Assume therefore that $z$ and $t_{0}$ are the only $t$-bad vertices in $G-\left\{u, u_{1}, u_{2}\right\}$, and so $X=\left\{u, u_{1}, u_{2}, t, z, t_{0}\right\}$.

If $u_{1} t_{0}$ is not an external edge of $G$, there exists a facial triangle $u_{1} t_{0} x$, with $x \neq z$, $x \neq t$ (recall that $u_{1}$ is not adjacent to $t$ ) and $x \neq u_{2}$ (recall that $u_{1}$ is an external vertex). In this case we define $D=X \backslash\left\{u_{1}, t_{0}\right\}=\left\{u_{2}, u, t, z\right\}$. Since every vertex of $X-D$ is contained in a triangle of $G-D$ we find, by applying Lemma 3.7, that $G-D$ is a WNT. Since $D \subseteq N[u], G$ is reducible. It follows that $u_{1} t_{0}$ is an external edge. With an analogous argument we prove that $u_{1} u_{2}$ is an external edge. If $u_{2} t$ is not an external edge of $G$, then there is a facial triangle $u_{2} t x$, with $x \neq u$ and $x \neq u_{1}$ (recall that $u_{1}$ is not adjacent to $t$ ). If $x=t_{0}$, then $u_{2}$ and $t_{0}$ are adjacent in $G$. If $u_{2} t_{0}$ is an external edge of $G$, then all three edges $u_{1} u_{2}, u_{2} t_{0}$ and $t_{0} u_{1}$ are external edges of $G$, and therefore $B$ is a block of order 6 in $G$. Otherwise, if $u_{2} t_{0}$ is an internal edge, then there exists an $x \notin\left\{t, u_{1}\right\}$ such that $u_{2} t_{0} x$ is a facial triangle. In this case we define $D=X \backslash\left\{u_{2}, t_{0}\right\}$. Since $G-X$ is a WNT, also $G-D$ is a WNT. Moreover $D \subseteq N[u]$, so $G$ is reducible. If $x \neq t_{0}$, then define $D=\left\{u, u_{1}, z, t_{0}\right\}$. Since $u_{2}$ and $t$ are contained in a triangle of $G-D$, we find that $G-D$ is a WNT. Since $D \subseteq N\left[u_{1}\right]$, we find that $G$ is reducible. Therefore we may assume that $u_{2} t$ is also an external edge of $G$. Finally we argue that $t_{0} t$ is an external edge of $G$. If not, then there is a facial triangle $t t_{0} x$, with $x \neq z$. If $x=u_{2}$, then $u_{2} t$ is not an external edge, contradicting the above assumption. Otherwise $x \neq u_{2}$ and $x \neq u_{1}$. So we define $D=X \backslash\left\{t, t_{0}\right\}$ and argue that $G-D$ is a WNT by refering to Lemma 3.7. Altogether, we proved that $u_{1} u_{2}, u_{2} t, t t_{0}, t_{0} u_{1}$ are external edges of $G$, therefore $B$ contains $u, u_{1}, u_{2}, t, z, t_{0}$ and no other vertices, hence $B$ is a block of order 6 .

Case $(b)$. Now we discuss the case when $t_{0}$ and $u$ are adjacent in $G$ (see Fig. 3 , Case $(b))$. If there is a vertex in the interior of $u_{1} u t_{0}$, then there is exactly one such vertex, for otherwise either $t_{0}$ is contained in a triangle of $G-\left\{u, u_{1}, u_{2}, t\right\}$ (and so $t_{0}$ is not a $t$-bad vertex) or there is a triangle $u_{1} z z^{\prime}$, where $z$ and $z^{\prime}$ are in the interior of $u_{1} u t_{0}$ (note that in this case $z$ and $z^{\prime}$ are not adjacent to $t$ ). If the latter happens, then let $T$ be the set of all $t$-bad vertices in $G-\left\{u, u_{1}, u_{2}\right\}$ and define $D=\left(\left\{u, u_{2}, t\right\} \cup T\right)$. Since $u_{1} z z^{\prime}$ is a triangle in $G-D$, we find that $G-D$ is a WNT (by Lemma 3.7). Since $D \subseteq N[t]$, we find that $G$ is reducible. If there is exactly one vertex in the interior of $u_{1} u t_{0}$, then this vertex is a $u_{1}$-bad vertex in $G-u$, and so $u_{1}$ is problematic in $G-u$. This case is treated in Section 4.4.2. Assume therefore that there is no vertex in the interior of $u_{1} u t_{0}$, so we have Case $(b)$ of Fig. 3 , where $B$ potentially contains some vertices of $G$.

Suppose that $t_{0}$ is the only $t$-bad vertex in $G-\left\{u, u_{1}, u_{2}\right\}$. In this case let $D=$ $\left\{u, u_{1}, u_{2}, t, t_{0}\right\}$. By Lemma 3.5, $G-D$ is a WNT. Since $D \subseteq N[u], G$ is reducible. Assume therefore that there are at least two $t$-bad vertices in $G-\left\{u, u_{1}, u_{2}\right\}$. 
If $t_{0} u_{1}$ is not an external edge of $G$, then there is a facial triangle $t_{0} u_{1} x$, with $x \neq u$ and $x \neq t$ (recall that $u_{1}$ is not adjacent to $t$ ) and $x \neq u_{2}$ (recall that $u_{1}$ is an external vertex). If $x$ is a $t$-bad vertex in $G-\left\{u, u_{1}, u_{2}\right\}$ then we get case (a) of Fig. 3. Assume therefore that $x$ is not a $t$-bad vertex in $G-\left\{u, u_{1}, u_{2}\right\}$. Let $T$ be the set of all $t$-bad vertices in $G-\left\{u, u_{1}, u_{2}\right\}, X=\left\{u, u_{1}, u_{2}, t\right\} \cup T$ and $D=X \backslash\left\{u_{1}, t_{0}\right\}$. By Lemma 3.5 we find that $G-X$ is a WNT, and therefore, by applying Lemma 3.7 we see that $G-D$ is a WNT. Reducibility of $G$ follows from $D \subseteq N[t]$ and $|D| \geq 4$. We conclude that $u_{1} t_{0}$ is an external edge of $G$.

If $u_{1} u_{2}$ is not an external edge of $G$, then there is a facial triangle $u_{1} u_{2} x$, with $x \neq u$, $x \neq t$ and $x \neq t_{0}$. Moreover $x$ is not adjacent to $t$ because $u_{1}$ and $u_{2}$ are external vertices of $G$. In this case we define the reduction by $D=X \backslash\left\{u_{1}, u_{2}\right\}$. This proves that both edges $t_{0} u_{1}$ and $u_{1} u_{2}$ are external edges of $G$. It follows that $\operatorname{deg}_{B}\left(u_{1}\right)=3$, and so $u_{1}$ is contained in a triangle $u_{1} a b$, with $a, b \notin B$ (see the assumption at the end of Section 4.4). Observe that $a$ and $b$ are not adjacent to $t$ (because $t \in B$ ). We define the reduction by $D=X \backslash\left\{u_{1}\right\}$. Since $u_{1}$ is contained in a triangle of $G-D$ we see that $G-D$ is a WNT, and so the reduction is well defined.

\subsection{2 $u_{1}$ is problematic in $G-u$.}

Note that there are no $u$-bad vertices in $G$, and so every vertex of $G-u$ is contained in a triangle of $G-u$.

Suppose that $u_{1}$ is problematic in $G-u$. If there are at least two $u_{1}$-bad vertices in $G-u$, then let $T$ be the set of all $u_{1}$-bad vertices in $G-u$, and define $D=\left\{u, u_{1}\right\} \cup T$. Since every vertex of $G-u$ is contained in a triangle of $G-u$, we find that every $u_{1}$-bad vertex in $G-u$ is adjacent to $u_{1}$. By Corollary 3.4 every bounded face of $G-D$ is triangular. Moreover, removing $u_{1}$ and all $u_{1}$-bad vertices from $G-u$ produces a graph in which all vertices are contained in a triangle. Therefore $G-D$ is a WNT, and since $D \subseteq N\left[u_{1}\right]$ and $|D| \geq 4, G$ is reducible. Assume therefore that there is exactly one $u_{1}$-bad vertex $x$ in $G-u$, and observe that the same arguments as above prove that $G-\left\{u, u_{1}, x\right\}$ is a WNT. Since every vertex of $G-u$ (and in particular $x$ ) is contained in a facial triangle of $G$ not containing $u$ (see the assumption at the end of Section 4.4), there exists a facial triangle $u_{1} x t$ in $G$, where $t \neq u$. (since $x$ is a $u_{1}$-bad vertex in $G-u$, both vertices $u_{1}$ and $x$ are contained in the same facial triangle, and removing $u_{1}$ breaks this facial triangle).

First let us prove that $u$ and $t$ are not adjacent. If they are adjacent, then let $T$ be the (possibly empty) set of all $t$-bad vertices in $G-\left\{u, u_{1}, x\right\}$ and define $D=\left\{u, u_{1}, x, t\right\} \cup T$. Since $D \subseteq N[t]$ and $G-D$ is a WNT we find that $G$ is reducible. This proves that $u$ is not adjacent to $t$. If $t$ is not problematic in $G-\left\{u, u_{1}, x\right\}$, then let $D=\left\{u, u_{1}, x, t\right\}$. By the defintion (of a problematic vertex) $G-D$ is a WNT, moreover $D \subseteq N\left[u_{1}\right]$, so $G$ is reducible. Assume therefore that $t$ is problematic in $G-\left\{u, u_{1}, x\right\}$. First we will assume that there is a $t$-bad vertex $t_{0}$ in $G-\left\{u, u_{1}, x\right\}$ adjacent to $u$.

\section{Case 1: A $t$-bad vertex is adjacent to $u$.}

There is at most one vertex in the interior of the 4 -cycle $u u_{1} t t_{0}$ adjacent to $t_{0}$. If 
not, $t_{0}$ is contained in a triangle of $G-\left\{u, u_{1}, x, t\right\}$, and so $t_{0}$ is not a $t$-bad vertex in $G-\left\{u, u_{1}, x\right\}$. Similarly, there is at most one vertex in the interior of the 4-cycle $u u_{1} t t_{0}$ adjacent to $u$. If not, $u$ is contained in a triangle $u w_{1} w_{2}$, where both $w_{1}$ and $w_{2}$ are in the interior of $u u_{1} t t_{0}$. In this case let $T$ be the set of all $t$-bad vertices in $G-\left\{u, u_{1}, x\right\}$ and define $D=\left(\left\{u_{1}, x, t\right\} \cup T\right) \backslash\left\{w_{1}, w_{2}\right\}$. Since $\left\{u_{1}, x, t, t_{0}\right\} \in D$ we have $|D| \geq 4$, and since $G-\left(\left\{u, u_{1}, x, t\right\} \cup T\right)$ is a WNT, we find (by applying Lemma 3.7) that $G-D$ is a WNT. Therefore assume that either there is no vertex of $G$ in the interior of the 4-cycle $u u_{1} x t$ adjacent to $t_{0}$ and $u$, or there is exactly one vertex in the interior of $u u_{1} x t$ adjacent to $t_{0}$ and $u$ (and note that this must be the same vertex). Call this vertex (if it exists) $z$, and note that $u_{1} z u, u z t_{0}$ and $t z t_{0}$ are facial triangles in this case.

We claim that $t_{0}$ is the only $t$-bad vertex in $G-\left\{u, u_{1}, x\right\}$. Let us first exclude the possibility $|T| \geq 3$, where $T$ is the set of all $t$-bad vertices in $G-\left\{u, u_{1}, x\right\}$. The edge $u u_{1}$ is an internal edge of $G$, so there is a facial triangle $u u_{1} w$, with $w \neq t$. If $|T| \geq 3$, let $D=(\{x, t\} \cup T) \backslash\{w\} .|D| \geq 4$ and since $G-\left(\left\{u, u_{1}, x, t\right\} \cup T\right)$ is a WNT we find (by Lemma 3.7) that $G-D$ is a WNT. Since $D \subseteq N[t], G$ is reducible. This leaves us with the possibility $|T|=2$, to be excluded next.

Let $t_{0}$ and $y$ be the only $t$-bad vertices in $G-\left\{u, u_{1}, x\right\}$. If there is no vertex in the interior of $u u_{1} t t_{0}$, then $u_{1}$ and $t_{0}$ are adjacent (recall that $u$ and $t$ are not adjacent). Let $u u_{1} w$ be the facial triangle, with $w \neq t_{0}$. If $w=y$, then let $D=\left\{u, u_{1}, x, t, t_{0}, y\right\}$. Since $D \subseteq N\left[u_{1}\right], G$ is reducible. If $w \neq y$, the reduction is given by $D=\left\{x, t, t_{0}, y\right\}$. Since $w \neq t$ (recal that $t$ and $u$ are not adjacent), and $w \neq x$ (recall that $u_{1}$ is an external vertex of $G$ ) we find that $u u_{1} w$ is a triangle in $G-D$, and so $G-D$ is a WNT (by Lemma 3.7). Since $D \subseteq N[t], G$ is reducible. Suppose now that there is a vertex $z$ in the interior of $u u_{1} t t_{0}$ adjacent to $t_{0}, u, u_{1}$ and $t$. First assume that $z=y$. If $u_{1}$ and $t_{0}$ are adjacent, then define $D=\left\{u, u_{1}, x, t, t_{0}, y\right\}$. We have $D \subseteq N\left[u_{1}\right]$ and $G-D$ is a WNT, so $G$ is reducible. Now assume that $u_{1}$ and $t_{0}$ are not adjacent. In this case there is a facial triangle $u u_{1} w$, with $w \neq t_{0}$ and $w \neq y=z$. Also $w \neq x$ because $u_{1}$ is an external vertex of $G$, and $w \neq t$ since $u$ and $t$ are not adjacent. In this case we define $D=\left\{t, x, y, t_{0}\right\}$. Observe that $D \subseteq N[t]$ and that $u u_{1} w$ is a triangle in $G-D$, so $G-D$ is a WNT (by Lemma 3.7). This proves that $G$ is reducible. It remains to check the case $z \neq y$. In this case we have $D=\left\{x, t, t_{0}, y\right\}$, and so $u u_{1} z$ is a triangle in $G-D$. It follows that $G-D$ is a WNT (by Lemma 3.7). Reducibility of $G$ follows from $D \subseteq N[t]$. This proves that $t_{0}$ is the only $t$-bad vertex in $G-\left\{u, u_{1}, x\right\}$, as claimed.

If $u_{1}$ and $t_{0}$ are adjacent then $D=\left\{u, u_{1}, x, t, t_{0}\right\}$. Since $D \subseteq N\left[u_{1}\right]$, and $G-D$ is a WNT, $G$ is reducible. Suppose therefore that $u_{1}$ and $t_{0}$ are not adjacent. In this case there is a vertex $z$ is the interior of $u u_{1} t t_{0}$ adjacent to all four vertices of this 4-cycle. Since $u t_{0}$ is an internal edge, there is a facial triangle $u t_{0} w$, with $w \neq z$. We have $w \neq t$ (because $u$ is not adjacent to $t$ ), and $w \neq u_{1}$ (because $u_{1}$ and $t_{0}$ are not adjacent by the assumption) and $w \neq x$ (for otherwise $x$ is contained in the triangle $x t t_{0}$ in $G-\left\{u, u_{1}\right\}$, and so $x$ is not a $u_{1}$-bad vertex in $G-u$ ). If $z$ is not problematic in $G-\left\{u, u_{1}, x, t, t_{0}\right\}$ then $G-\left\{u, u_{1}, x, t, t_{0}, z\right\}$ is a WNT. Define $D=\left\{t, x, z, u_{1}\right\}$ and observe that $D \subseteq N[t]$. Since $u t_{0} w$ is a triangle in $G-D$ we find (by Lemma 3.7) that $G-D$ is a WNT. It remains to check the case when $z$ is problematic in 
$G-\left\{u, u_{1}, x, t, t_{0}\right\}$. Let $T$ be the set of all $z$-bad vertices in $G-\left\{u, u_{1}, x, t, t_{0}\right\}$. Define $D=\left\{z, u, t_{0}\right\} \cup T$. Since $G-\left\{u, u_{1}, x, t, t_{0}, z\right\} \cup T$ is a WNT, we find that $G-D$ is a WNT, because $u_{1} x t$ is a triangle in $G-D$. $G$ is reducible because $D \subseteq N[z]$.

\section{Case 2: No $t$-bad vertex is adjacent to $u$.}

Assume now that no $t$-bad vertex is adjacent to $u$. We will prove that $x$ and $u$ are not adjacent in $G$. For the purpose of a contradiction assume that $x$ and $u$ are adjacent. Then there are two possible drawings of the graph induced by $u, u_{1}, x, t$ : either the triangle $u_{1} x t$ lies in the interior of the triangle $u_{1} x u$, or it lies in the exterior. If the triangle $u_{1} x t$ lies in the interior of the triangle $u_{1} x u$ then the edge $x t$ is incident to a triangular face contained in the interior of the 4-cycle $u_{1} t x u$. Let $x t w$ be the triangle bounding this triangular face. Since there are no multiple edges in $G$ and $u$ is not adjacent to $t$, we find that $w \notin\left\{u, u_{1}\right\}$. It follows that $x$ is contained in a traingle of $G-\left\{u, u_{1}\right\}$, a contradiction (recall that $x$ is a $u_{1}$-bad vertex in $G-u$ ).

Let's consider the case when triangle $u_{1} x t$ lies in the exterior of the triangle $u_{1} x u$. We claim that, the interior of $u u_{1} x$ contains at most one vertex of $G$. To see this let $w$ be a vertex in the interior of $u u_{1} x$ adjacent to $x$. The edge $x w$ is an internal edge, so it is incident to two facial triangles $x w y$ and $x w y^{\prime}$. If $y$ or $y^{\prime}$ is not $u$ or $u_{1}$, then $x$ is contained in a triangle of $G-\left\{u, u_{1}\right\}$, and so $x$ is not a $u_{1}$-bad vertex in $G-u$, a contradiction. Therefore $y=u_{1}$ and $y^{\prime}=u$. If there is a vertex of $G$ in the interior of the tirangle $u u_{1} w$, then $u$ is contained in a triangle of $G-\left(\left\{u_{1}, x, t\right\} \cup T\right)$, where $T$ is the set of all $t$-bad vertices in $G-\left\{u, u_{1}, x\right\}$. Define $D=\left\{u_{1}, x, t\right\} \cup T$. By Lemma 3.5. $G-\left(\left\{u, u_{1}, x, t\right\} \cup T\right)$ is a WNT, and therefore $G-D$ is a WNT by Lemma 3.7. This proves that there is no vertex in the interior of $u u_{1} w$, and so $w$ is the only vertex in the interior of triangle $u u_{1} x$, as claimed.

We now discuss both cases: there is a vertex in the interior of $u u_{1} x$, or there is no such vertex. Suppose that $w$ is (the only) vertex in the interior of triangle $u u_{1} x$. Then $w$ is a $u_{1}$-bad vertex in $G-u$, this contradicts that $x$ is the only $u_{1}$-bad vertex in $G-u$ (see assumptions in the second paragraph of Section 4.4.2). It remains to check the case when there is no vertex in the interior of $u u_{1} x$. In this case $u u_{1} x$ is a facial triangle, and if $\operatorname{deg}_{G}(u) \geq 4$, then $u$ is contained in a triangle of $G-\left(\left\{u_{1}, x, t\right\} \cup T\right)$, because $t$ is not adjacent to $u$, and no vertex in $T$ is adjacent to $u$ (and because $u$ is an internal vertex of $G)$. In this case we define $D=\left\{u_{1}, x, t\right\} \cup T$. Since $G-\left(\left\{u, u_{1}, x, t\right\} \cup T\right)$ is a WNT, we find by an application of Lemma 3.7 that $G-D$ is a WNT. Since $D \subseteq N[t]$, $G$ is reducible. It follows that $\operatorname{deg}_{G}(u)=3$, and let $w \neq u_{1}, x$ be the third neighbor of $u$ in $G$. Clearly, $w$ is adjacent to $x$ and $u_{1}$, because $u$ is an internal vertex and $G$ is a WNT. Let $T$ be the set of all $w$-bad vertices in $G-\left\{u, u_{1}, x\right\}$ (if any), and define $D=\left\{u, u_{1}, x, w\right\} \cup T$. By Lemma 3.5, $G-D$ is a WNT, and since $D \subseteq N[w]$, we conclude that $G$ is reducible. This proves that $u$ and $x$ are not adjacent in $G$.

Since no $t$-bad vertex in $G-\left\{u, u_{1}, x\right\}$ is adjacent to $u$ (and $u$ and $t$ are not adjacnet), we find that $u$ is contained in a triangle of $G-\left(\left\{u_{1}, x, t\right\} \cup T\right)$, where $T$ is the set of all $t$-bad vertices in $G-\left\{u, u_{1}, x\right\}$. We define $D=\left\{u_{1}, x, t\right\} \cup T$. Lemma 3.7 proves that $G-D$ is a WNT. Since $D \subseteq N[t]$, we find that $G$ is reducible. This completes the 
proof of Theorem 2.8.

Acknowledgement: The author greatly appreciates discussions with Uroš Milutinović while working on this paper. The author is supported by research grant P1-0297 of Ministry of Education of Slovenia.

\section{References}

[1] C.N. Campos, Y. Wakabayashi, On dominating sets of maximal outerplanar graphs, Discrete Appl. Math. 161 (2013), no. 3, 330-335.

[2] R. Diestel, Graph theory. Fourth edition. Graduate Texts in Mathematics, 173. Springer, Heidelberg, 2010.

[3] M. Dorfling, W. Goddard, M.A. Henning, Domination in planar graphs with small diameter II, Ars Combin. 78 (2006), 237-255.

[4] M. Furuya, N. Matsumoto, A note on the domination number of triangulations, J. Graph Theory 79 (2015), no. 2, 83-85.

[5] W. Goddard, M.A, Henning, Domination in planar graphs with small diameter, J. Graph Theory 40 (2002), no. 1, 1-25.

[6] T. Honjo, K. Kawarabayashi, A. Nakamoto, Dominating sets in triangulations on surfaces, J. Graph Theory 63 (2010), 17-30.

[7] E. King, M. Pelsmajer, Dominating sets in plane triangulations, Discrete Math., 310 (2010), 2221-2230

[8] Z. Li, E. Zhu, Z. Shao, J. Xu, Jin On dominating sets of maximal outerplanar and planar graphs, Discrete Appl. Math. 198 (2016), 164-169.

[9] H. Liu and M.J. Pelsmajer, Dominating sets in triangulations on surfaces, Ars Math Contemp. 4 (2011), 177-204.

[10] G. MacGillivray, K. Seyffarth, Domination numbers of planar graphs, J. Graph Theory 22 (1996), no. 3, 213-229.

[11] L.R. Matheson, R.E. Tarjan, Dominating sets in planar graphs, European J. Combin. 17 (1996), no. 6, 565-568.

[12] B. Mohar, C. Thomassen, Graphs on surfaces. Johns Hopkins Studies in the Mathematical Sciences. Johns Hopkins University Press, Baltimore, MD, 2001.

[13] M.D. Plummer, X. Zha, On certain spanning subgraphs of embeddings with applications to domination, Discrete Math., 309 (2009), 4784-4792.

[14] M.D. Plummer, D. Ye, X. Zha, Dominating plane triangulations. Discrete Appl. Math., 211 (2016), 175-182 
[15] S. Tokunaga, Dominating sets of maximal outerplanar graphs, Discrete Appl. Math. 161 (2013), 3097-3099. 